\title{
Carcinoma de conductos colectores de Bellini: recurrencia a mediano plazo
}

Juan Sebastián Rodríguez-Álvarez, ${ }^{1}$ José Cruz-Ruiz, ${ }^{1}$ Roberto Javier ReddingOchoa, ${ }^{2}$ Armando Gamboa-Domínguez, ${ }^{2}$ Daniel Montante-Montes de Oca, ${ }^{2}$ Francisco Rodríguez-Covarrubias ${ }^{1}$

\section{Resumen}

CASO CLÍNICO: Paciente femenina de 77 años de edad, que inició su cuadro clínico con dolor en el flanco izquierdo, de 20 días de evolución, además de dolor óseo. El ultrasonido reportó una masa dependiente del polo superior del riñón izquierdo. La tomografía simple, complementada con resonancia magnética, evidenció una lesión de $5.7 \times 7.3 \mathrm{~cm}$, de consistencia sólida, con necrosis central y extensión hacia el seno renal (T2a; GII). El tratamiento consistió en nefrectomía radical laparoscópica izquierda. Se estableció el diagnóstico de carcinoma de conductos colectores de Bellini. El seguimiento a 5 meses mostró un nuevo ganglio latero-aórtico izquierdo de $16 \mathrm{~mm}$. Se realizó escisión de la lesión y linfadenectomía retroperitoneal, que reportó 20 ganglios con hiperplasia linfoide negativa a malignidad. Treinta y nueve meses después de la nefrectomía, la tomografía computada evidenció dos tumores retroperitoneales: uno paraaórtico y otro intercavoaórtico, compatibles con recurrencia de la enfermedad. Se llevó a cabo metastasectomía, que histopatológicamente reportó un tumor sólido, irregular, de color blanco-grisáceo, con áreas extensas de hemorragia en su porción inferior izquierda y focos discretos de necrosis. Hoy día, después de 42 meses de seguimiento, la paciente se mantiene en vigilancia y sin datos de recurrencia.

CONCLUSIONES: El carcinoma de conductos colectores es una neoplasia excepcional. El caso aquí reportado corresponde al de mayor supervivencia y tiempo de recurrencia descrito en un caso de T2M0 sintomático: 39 meses.

PALABRAS CLAVE: Carcinoma de conductos colectores; recurrencia; supervivencia.

Rev Mex Urol. 2018 March-April;78(2):149-154.

\section{Bellini duct carcinoma:}

\section{Medium-term recurrence}

Juan Sebastián Rodríguez-Álvarez, ${ }^{1}$ José Cruz-Ruiz, ${ }^{1}$ Roberto Javier ReddingOchoa, ${ }^{2}$ Armando Gamboa-Domínguez, ${ }^{2}$ Daniel Montante-Montes de Oca, ${ }^{2}$ Francisco Rodríguez-Covarrubias ${ }^{1}$

\section{Abstract}

CLINICAL CASE: A 77-year-old woman had clinical symptom onset with pain in the left flank of 20-day progression and bone pain. An
${ }^{1}$ Departamento de Urología.

${ }^{2}$ Departamento de Patología.

Instituto Nacional de Ciencias Médicas y Nutrición Salvador Zubirán, Ciudad de México.

Recibido: octubre 2017

Aceptado: marzo 2018

Correspondencia

Juan Sebastián Rodríguez Álvarez

sebastian.alvarez.pi@gmail.com

Este artículo debe citarse como

Rodríguez-Álvarez JS, Cruz-Ruiz J, Redding-Ochoa J, Gamboa-Domínguez A, Montante-Montes de Oca D, Rodríguez-Covarrubias F. Carcinoma de conductos colectores de Bellini: recurrencia a mediano plazo. Rev Mex Urol. 2018 marzo-abril;78(2):149-154. DOI: https://doi.org/10.24245/revmexurol.v78i2.1677 
ultrasound study identified an upper pole-dependent mass on the left kidney. A non-contrast-enhanced tomography scan, complemented with a magnetic resonance study, revealed a solid lesion with central necrosis that extended into the renal sinus (T2a; GII). Treatment was laparoscopic left radical nephrectomy. The diagnosis was Bellini duct carcinoma. A new 16-mm left lateroaortic lymph node was found at the follow-up at 5 months. The lesion was excised, and retroperitoneal lymph node dissection was carried out, producing 20 lymph nodes with lymphoid hyperplasia that were negative for malignancy. Thirtynine months after the nephrectomy, a computed tomography scan identified a retroperitoneal paraaortic tumor and an intercaval-aortic retroperitoneal tumor consistent with disease recurrence. Metastasectomy was performed and the histopathologic report described a solid, irregular, greyish-white tumor that had extensive hemorrhaging in the left lower portion and a moderate amount of necrotic foci. At present, after 42 months of follow-up, the patient is under surveillance and has no signs of recurrence.

CONCLUSIONS: Collecting duct carcinoma is a rare neoplasia. The case reported herein has the longest survival period and recurrence time presently described for symptomatic T2M0 disease: 39 months.

KEYWORDS: Collecting duct carcinoma; Recurrence; Survival.

\section{ANTECEDENTES}

El carcinoma de conductos colectores es un tumor renal poco frecuente descrito en 1979. ${ }^{1}$ Según la cohorte más grande reportada por la National Cancer Database (NCDB), se estimó una incidencia de $0.2 \%$ y supervivencia media de 13.2 meses. ${ }^{2}$ Su histogénesis corresponde a células de los conductos colectores en la médula renal. De acuerdo con su origen embriológico, el carcinoma de los conductos colectores se inicia en la yema ureteral y el carcinoma medular en el blastema metanéfrico.

El 90\% de los pacientes manifiesta síntomas y enfermedad a distancia similares a los que provoca el cáncer renal, además de pérdida de peso. El diagnóstico se establece después de analizar la pieza de la nefrectomía; sin embargo, algunos autores recomiendan obtener una biopsia previa al procedimiento quirúrgico, debido al alto riesgo de mortalidad perioperatoria. ${ }^{3,4}$

\section{CASO CLÍNICO}

Paciente femenina de 77 años de edad, con antecedente de diabetes tipo 2, nefropatía secundaria (KDIGO 3A) e hipertensión arterial sistémica de larga evolución, sin complicaciones. Inició su cuadro clínico con dolor en el flanco izquierdo, de 20 días de evolución, además de dolor óseo. Después de la exploración física se solicitó un ultrasonido, que reportó una masa dependiente del polo superior del riñón izquierdo. La tomografía simple, complementada con resonancia magnética, evidenció una lesión de 5.7 × 7.3 $\mathrm{cm}$, de consistencia sólida, con necrosis central y extensión hacia el seno renal (T2a; GII) (Figura 1). Se descartó metástasis a hueso por gamagrafía ósea. Los estudios de laboratorio de ingreso documentaron anemia microcítica hipocrómica.

El tratamiento consistió en nefrectomía radical laparoscópica izquierda, cuya pieza histopatológica reportó un tumor de $9 \mathrm{~cm}$, que afectaba 
Rodríguez-Álvarez JS y col. Carcinoma de conductos colectores de Bellini

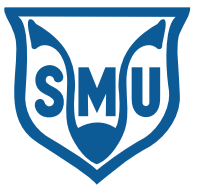

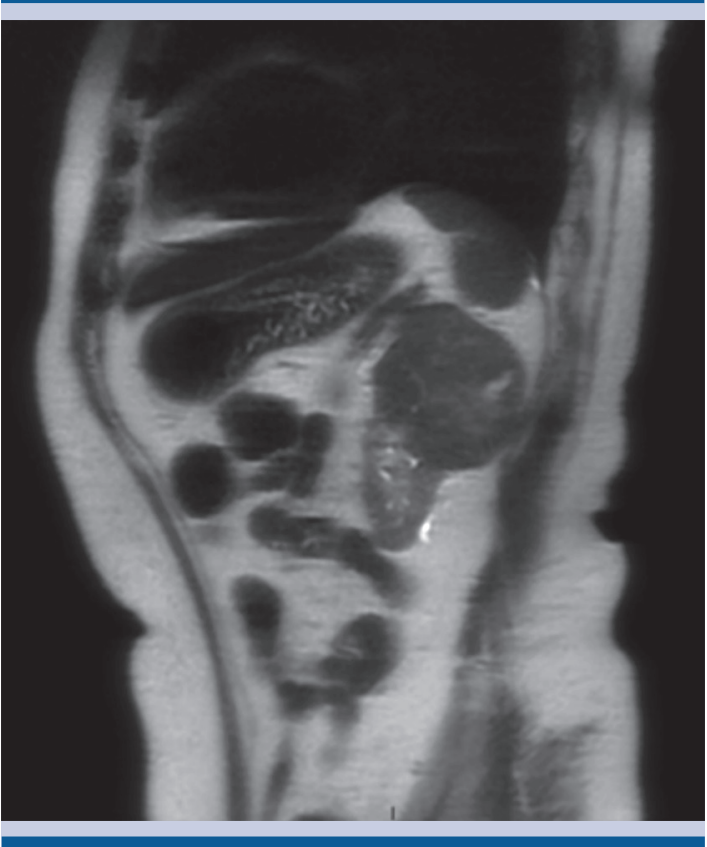

Figura 1. Resonancia magnética. Corte sagital que muestra una lesión sólida en el riñón izquierdo, con necrosis central.

el polo superior y medio del riñón izquierdo. La neoplasia mostró superficies blancas, sólidas y multinodulares sin invasión del seno renal; microscópicamente se observó una neoplasia epitelial maligna, formada por túbulos de luces irregulares bordeadas por células con marcado pleomorfismo celular y nuclear (células en "estoperol") con márgenes negativos. Los resultados fueron sugerentes de carcinoma de conductos colectores de Bellini (Figura 2). Durante el seguimiento, a 5 meses, la tomografía computada simple mostró un nuevo ganglio latero-aórtico izquierdo de $16 \mathrm{~mm}$, cercano al lecho quirúrgico. Por sospecha de recurrencia se realizó escisión de la lesión, además de linfadenectomía retroperitoneal abierta izquierda que reportó la coexistencia de 20 ganglios con hiperplasia linfoide negativa a malignidad. La paciente se mantuvo estable y en vigilancia. La tomografía computada documentó probable linfocele en el
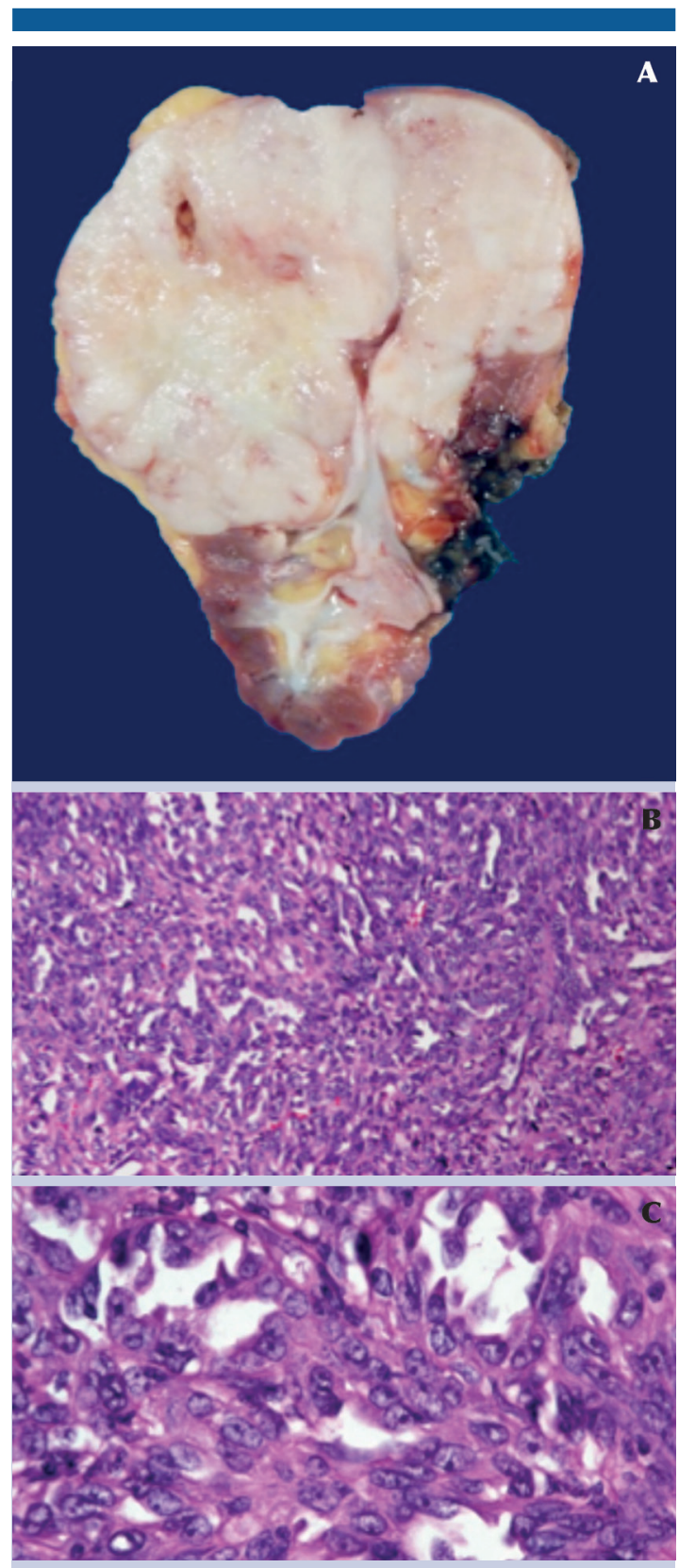

Figura 2. Tumor de $9 \mathrm{~cm}$ en el polo superior y medio del riñón izquierdo con superficies blancas, sólidas y multinodulares sin afectación del seno renal (A). Microscopia que muestra túbulos de luces irregulares bordeadas por células con marcado pleomorfismo celular y nuclear (células en forma de "estoperol") (B y C). 
lecho quirúrgico, que permaneció en vigilancia estrecha. Treinta y nueve meses después de la nefrectomía, la tomografía computada evidenció dos tumores retroperitoneales: uno paraaórtico y otro intercavoaórtico, con ejes mayores de 4 y $5 \mathrm{~cm}$, respectivamente, compatibles con recurrencia de la enfermedad (Figura 3 ).

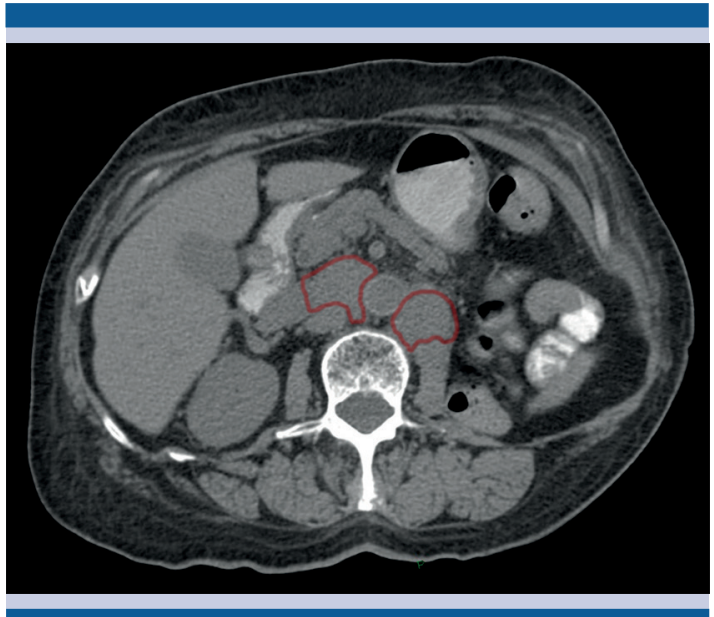

Figura 3. Tomografía computada. Corte axial que evidencia dos lesiones (rojo) en el espacio intercavoaórtico y paraaórtico, de 7 y $5 \mathrm{~cm}$, respectivamente.

Se llevó a cabo metastasectomía, que histopatológicamente reportó un tumor sólido, irregular, de color blanco-grisáceo, con áreas extensas de hemorragia en su porción inferior izquierda y focos discretos de necrosis (Figura 4).

Hoy día, después de 42 meses de seguimiento, la paciente permanece en vigilancia y sin datos de recurrencia. Microscópicamente, con los objetivos de bajo aumento, se observó un patrón de crecimiento con cordones entrecruzados, con espacios vacíos angulados, en forma de hendiduras o rendijas; con los objetivos de mayor aumento las células neoplásicas mostraron pérdida de la polaridad, algunas de ellas parecidas a la forma de un "estoperol", compatible con recurrencia de carcinoma renal de conductos colectores

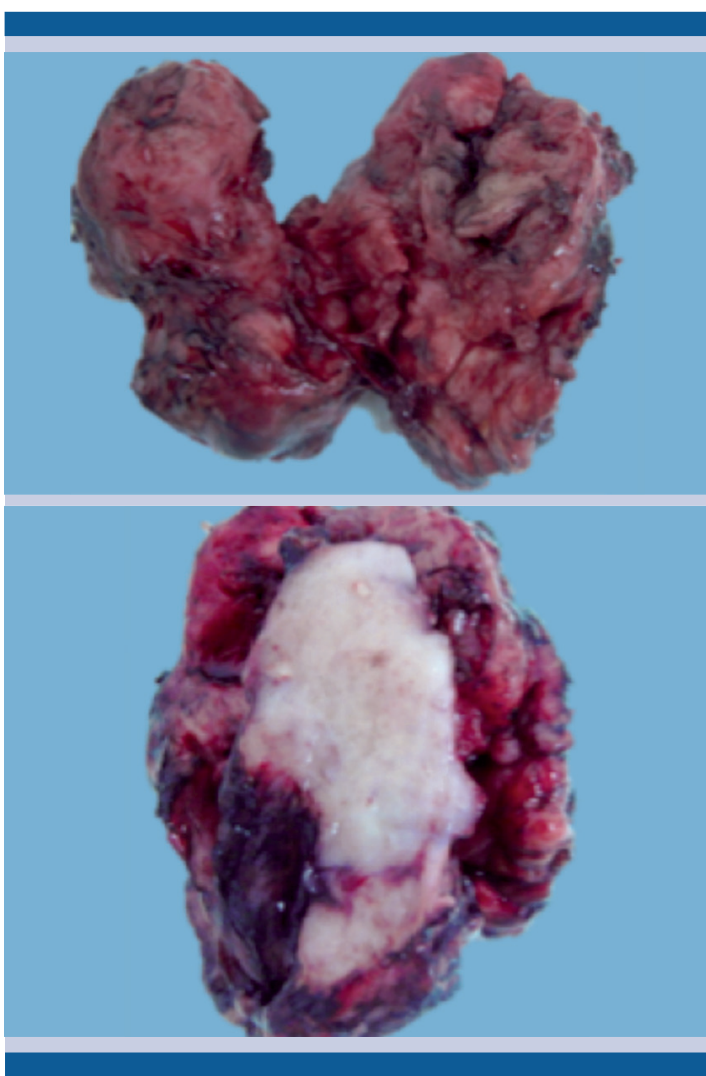

Figura 4. Tumor irregular, de color blanco-grisáceo, con áreas de hemorragia y necrosis.

(Figura 5). Los núcleos se observaron grandes y ovales; cromatina abierta y rechazada a la periferia y nucléolo prominente. La inmunorreacción resultó positiva para PAX8 y negativa para p63. La paciente tuvo evolución clínica favorable y egresó estable.

\section{DISCUSIÓN}

El carcinoma de conductos colectores es una de las neoplasias renales más raras, cuyas manifestaciones y diagnóstico suelen establecerse en estadios avanzados de la enfermedad. La función de la quimioterapia, radioterapia e inmunoterapia ha demostrado beneficios limitados y sujetos a discusión. ${ }^{5}$ Sólo un estudio retrospectivo ha reportado disminución de la mortalidad cuando 
Rodríguez-Álvarez JS y col. Carcinoma de conductos colectores de Bellini
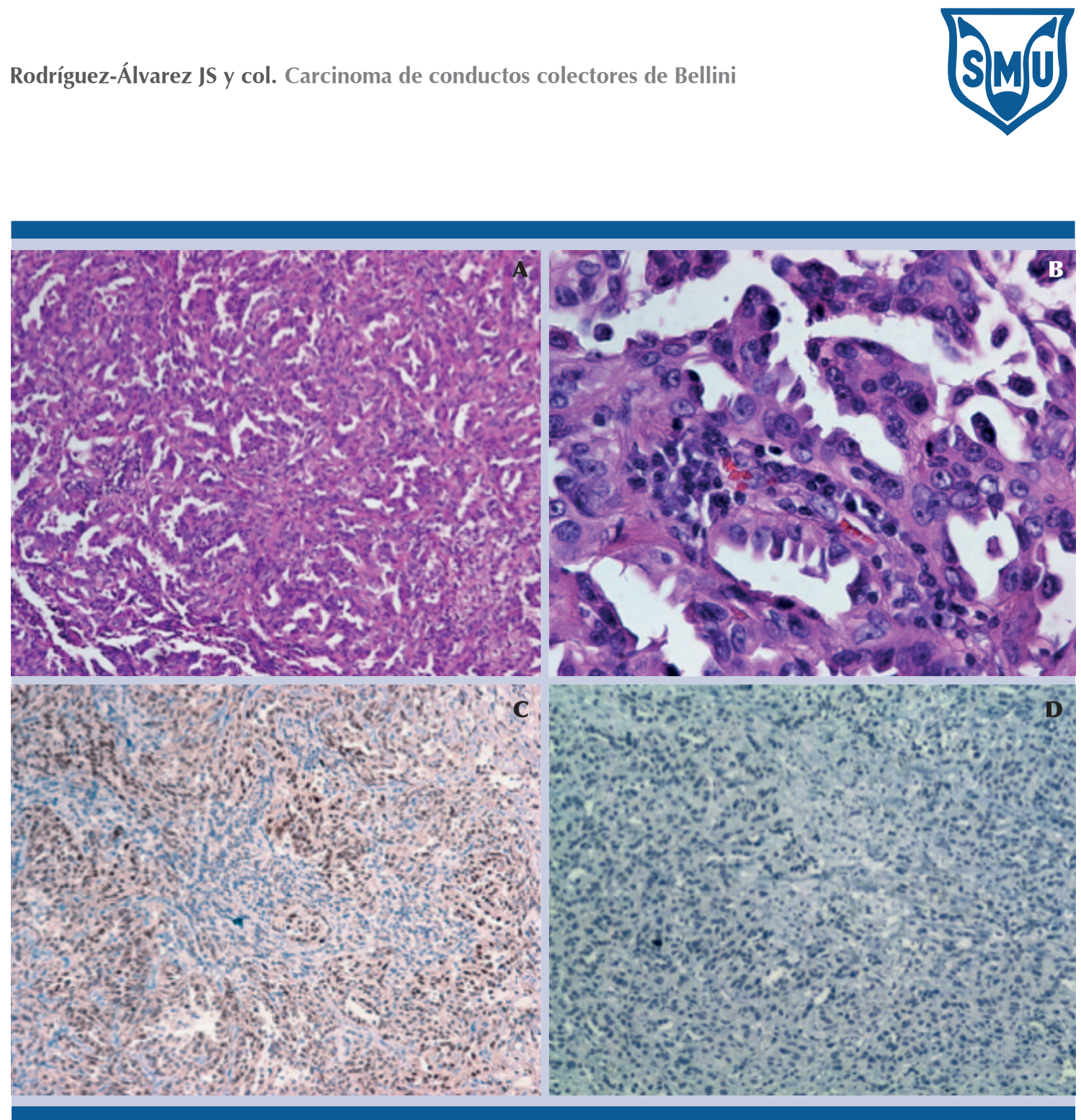

Figura 5. Patrón de crecimiento con cordones que se entrecruzan, dejando espacios vacíos angulados (A); células neoplásicas con pérdida de la polaridad, algunas muestran la forma característica de una "tachuela" o "estoperol" (B); inmunorreacción positiva para PAX8 (C) y negativa para p63 (D).

se complementa con cirugía en pacientes con metástasis. ${ }^{2}$

Puesto que se trata de un tumor excepcional y el diagnóstico se establece en etapas avanzadas, le confiere un mal pronóstico. Un estudio de 41 casos pareados con carcinoma de células claras reportó una tasa de supervivencia similar a la de pacientes con carcinoma de conductos colectores de Bellini. ${ }^{6}$
Los sitios de metástasis más frecuentes son los nodos, las glándulas adrenales, el hueso, pulmón e hígado. ${ }^{1}$ El pronóstico de supervivencia varía de 11 a 15 meses; sin embargo, dos estudios señalan un caso de 58 (pT1) y otro de 120 meses (T2) con diagnóstico fortuito de la enfermedad. De acuerdo con la bibliografía revisada, el caso aquí reportado corresponde al de mayor supervivencia y tiempo de recurrencia descrito en un caso de T2M0 sintomático: 39 meses. $^{7}$ 
La media de recurrencia local es de 4.9 meses (límite máximo de 10 meses). ${ }^{3,8}$ En la paciente de este estudio se reportó una recurrencia local de 39 meses, con adecuado control oncológico mediante resección quirúrgica.

\section{REFERENCIAS}

1. Yoon SK, Rha SH. Collecting duct carcinoma. En: Guermazi A, Editor. Imaging in kidney cancer. 1aㅡ ed. Berlin: SpringerVerlag, 2003; 171-185.

2. Sui W, Matulay JT, Robbins DJ, James MB, Onyeji IC, et al. Collecting duct carcinoma of the kidney: Disease characteristics and treatment outcomes from the National Cancer Database. Urol Oncol 2017;35(9):540.e13-18.

3. Ciszewski S, Jakimow A, Smolska-Ciszewska B. Collecting (Bellini) duct carcinoma: A clinical study of a rare tumour and review of literatura. Can Urol Assoc J 2015;9(910):E589-93.

4. Mishra AK, Manikandan R, Dorairajan LN, Mittal JK, Rekha JS. Bellini duct carcinoma: a rare entity J Clin Diag Res 2016;10(10):PD01-02.

5. Dason S, Allard C, Sheridan-Jonah A, Gill J, Jamshaid H, et al. Management of renal collecting duct carcinoma: a systematic review and the McMaster experience. Curr Oncol 2013;20:223-232.

6. Karakiewicz PI, Trinh QD, Rioux-Leclercq N, de la Taille A, Novara G, et al. Collecting duct renal cell carcinoma: a matched analysis of 41 cases. Eur Urol 2007;52:1140-5. doi: 10.1016/j.eururo.2007.01.070

7. Husillos A, Herrans-Amo F, Subirá D, Lledó E, et al Collecting duct renal cell carcinoma Actas Urol Esp 2011;35:368-71.

8. Rodríguez-Carlin JA, Vidal-Mora I, Arellano L, Castillo OA. Carcinoma renal de los conductos colectores de Bellini con recidiva retroperitoneal por otro tipo histológico de tumor renal. Arch Esp Urol 2013;66(6):597-1.

\section{AVISO IMPORTANTE}

La Revista Mexicana de Urología se convierte en una publicación solo digital, con todas las ventajas que los medios y dispositivos electrónicos ofrecen. Usted podrá revisar la información mediante el sitio web (www.revistamexicanadeurologia.org.mx) o descargando la app para Android o iPhone.

Para consultar el texto completo de los artículos deberá registrarse por una sola vez con su correo electrónico, crear una contraseña, indicar su nombre, apellidos y especialidad.

Esta información es indispensable para saber qué consulta y cuáles son sus intereses, y poder en el futuro inmediato satisfacer sus necesidades de información. 